\title{
COMPONENTES DO PROCESSO DE PASTEJO DE CORDEIROS EM AZEVÉM SOB DIFERENTES INTENSIDADES E MÉTODOS
}

\author{
COMPONENTS OF LAMB GRAZING PROCESS ON RYEGRASS \\ (LOLIUM MULTIFLORUM L.) UNDER DIFFERENT INTENSITIES AND METHODS
}

\author{
Barbosa, C.M.P. ${ }^{1 *}$, Carvalho, P.C.F. ${ }^{1}$, Cauduro, G.F. ${ }^{1}$, Lunardi, R. ${ }^{1}$, Gonçalves, E.N. ${ }^{1}$ \\ e Devincenzi, T. ${ }^{1}$
}

${ }^{1}$ Universidade Federal do Rio Grande do Sul. Faculdade de Agronomia. Departamento de Plantas Forrageiras e Agrometeorologia. Av. Bento Gonçalves, 7712. CEP 91540-000. Porto Alegre/RS. Brasil. *cristinampb@yahoo.com.br

\section{PALAVRAS CHAVE ADICIONAIS}

Comportamento ingestivo. Tempo de pastejo. Ruminação.

\section{RESUMO}

O objetivo foi avaliar variáveis que compõem o processo de pastejo de cordeiros em pastagens de azevém anual (Lolium multiflorum Lam.). Os tratamentos foram duas intensidades de pastejo moderada e baixa (oferta de forragem 2,5 ou 5,0 vezes o potencial de consumo dos animais), em pastejo com lotação contínua ou rotacionada, com carga variável. O delineamento experimental foi o de blocos casualizados, em um esquema fatorial ( 2 intensidades $\times 2$ métodos $\times 3$ repetições). Não houve interação entre os métodos e as intensidades e seus efeitos foram analisados de forma independente. As ofertas de forragem (OF) não foram diferentes $(p=0,3393)$ entre os métodos de pastejo, garantindo, assim, a condição fundamental para comparação entre os tratamentos. As massas de forragem (MF) foram: 3159 e $2327 \mathrm{~kg}$ de MS/ha, para os métodos de pastejo de lotação rotacionada e contínua, respectivamente; 1971 e $3514 \mathrm{~kg}$ de MS/ha, para as intensidades de pastejo moderada e baixa, respectivamente. Não houve diferenças significativas para número de refeições (NR), tempo de duração das refeições (TRf), número de intervalos entre refeições (IR), duração média do intervalo (DMI), tempo de pastejo (TP), de ruminação (TR) e gasto para outras atividades (TO) $(p>0,1)$ em relação a métodos de pastejo. Não houve diferença estatística para TR, NR, TRf e IR $(p>0,1)$ para as diferentes intensidades de pastejo. DMI, TP, TO, TB e TEA foram influenciados pelas

Recibido: 20-8-07. Aceptado: 6-5-08.

\section{AdDitional KEYWORDS}

Foraging behaviour. Grazing time. Rumination.

intensidades de pastejo $(p<0,1)$. Efeito de métodos de pastejo $(p<0,1)$ foi observado apenas para TB e TEA, devido a maior MF na lotação rotacionada. A manutenção de intensidades de pastejo adequadas aos animais, mais do que a escolha entre métodos de pastejo mostrou ser de extrema importância, no que diz respeito à construção de ambientes pastoris sustentáveis para a produção de cordeiros de alta qualidade.

\section{SUMMARY}

The aim of this study was to evaluate the components of lamb grazing process in ryegrass pastures (Lolium multiflorum Lam.). The treatments were two grazing intensities: moderate and low (forage suply: 2.5 and 5 -fold the animal intake potential, respectively) in rotational and continuous stocking. The experimental design was a randomized complete block arranged in a factorial with three replicates ( 2 intensities $x 2$ methods $x$ 3 replicates). There was no interaction among grazing intensities and methods, and its effects were analyzed separately. There was no difference of herbage allowance between grazing methods ( $p=0.3393)$, and so, the basic conditions to compare all treatments were reached. Herbage mass during the experiment were: 3159 and 2327 $\mathrm{kg}$ of DM/ha for rotational and continuous stocking; and 1971 and $3514 \mathrm{~kg}$ of DM/ha for moderate and 
low grazing intensities, respectively. There was no significant differences for number of meals (M), meal duration (MD), inter meals intervals (IMI), duration of intervals (DI), grazing time (GT), ruminating time (RT) and idling time $(I T)(p>0.1)$ for grazing methods. There was no statistical difference for RT, M, MD and IMI ( $p>0.1)$ regarding grazing intensities. DI, GT, IT, bite rate (BR) and time for feeding station (TFS) were affected by grazing intensity $(p<0.1)$. Grazing methods affected just BR and TFS $(p<0.1)$ due to the higher herbage mass under rotational stocking. The use of correct grazing intensities for the animals, more than the choice between grazing methods, is capital to built sustainable grazing environments aiming the production of high quality lambs.

\section{INTRODUÇÃO}

O manejo da pastagem é a arte de criar ambientes pastoris adequados à obtenção dos nutrientes requeridos pelo animal, por meio da otimização de sua velocidade de ingestão (Carvalho et al., 2005). Neste sentido, existem ferramentas de gerenciamento da estrutura do pasto que permitem otimizar o pastejo no ambiente pastoril (i.e., altura, métodos e intensidades de pastejo, oferta de forragem).

Entender os fatores que controlam o número e a duração das refeições, e o tempo da estação alimentar, em relação ao estado do pasto, são passos importantes para predizer a aquisição de nutrientes pelos animais em pastejo (Baumont et al., 2004). Esses fatores afetam o desempenho animal e são de extrema importância, principalmente no que se refere à construção de um ambiente pastoril adequado para a produção de carne de cordeiro de alta qualidade (Carvalho, 2005). No entanto, essa concepção contraria os objetivos precípuos de antigos modelos de pesquisa, em que a prioridade é comumente focada na produtividade dos sistemas, enquanto que aspectos como comportamento animal, qualidade do produto final e questões ambientais são totalmente negligenciadas.

Segundo Carvalho e Moraes (2005), a qualidade de um ambiente pastoril adequado do ponto de vista alimentar (ingestão de nutrientes), pode ser medida pelo seguinte conjunto de respostas comportamentais: (i) em situações de elevado nível de alimentação os animais escolhem poucas estações alimentares enquanto passam bastante tempo explorando-as; (ii) o deslocamento entre as estações alimentares pode ser longo; (iii) o número de refeições é maior e a duração das refeições é menor; (iv) o intervalo entre refeições é maior; (v) menor é o tempo de pastejo.

Na busca por este ambiente pastoril de qualidade, as principais ferramentas disponíveis, e passíveis de uso para gerenciamento da estrutura do pasto, em curto prazo, seriam a manipulação da intensidade de pastejo, da adubação e do método de pastejo. Diferentes intensidades e métodos de pastejo definem a quantidade e a forma com que a forragem será disponibilizada ao animal. Assim sendo, os objetivos desse estudo com cordeiros, pastejando azevém manejado em diferentes métodos (lotação contínua e rotacionada) e intensidades de pastejo (moderada e baixa) foram: (i) comparar os diferentes métodos e intensidades de pastejo por meio de variáveis associadas ao processo de ingestão de forragem; (ii) estudar os mecanismos de adaptação do comportamento ingestivo de cordeiros, em face de diferentes quantidades e formas de apresentação do alimento para o animal; (iii) definir ambientes de pastejo adequados para a produção de cordeiros de alta qualidade.

\section{MATERIALEMÉTODOS}

O experimento foi conduzido na fazenda experimental da Universidade Federal do Rio Grande do Sul, UFRGS, no município de Eldorado do Sul/RS, a 3005'22"S, 51³9'08"W e $46 \mathrm{~m}$ de altitude. A pastagem de azevém (Lolium multiflorum Lam, cv. Comum) foi semeada em abril de 2003, em sistema de plantio direto. A vegetação da área foi previamente dessecada, utilizando-se herbici-

Archivos de zootecnia vol. 59, núm. 225, p. 40. 
da de princípio ativo glifosate e fertilizada com $1 \mathrm{t} \mathrm{ha}^{-1}$ de calcário e $200 \mathrm{~kg} \mathrm{ha}^{-1}$ da fórmula 5-20-20. Foram realizadas duas aplicações de adubo nitrogenado: $75 \mathrm{~kg}$ de $\mathrm{Nha}^{-1}$ em maio e $75 \mathrm{~kg}$ de $\mathrm{Nha}^{-1}$ em setembro.

$\mathrm{O}$ ensaio foi realizado seguindo um delineamento de blocos casualizados, em um esquema fatorial ( $2 \times 2$ ), com 3 repetições, correspondendo a 12 unidades experimentais, com uma área variável de 0,23 a 0,41 ha.

Utilizou-se cordeiros de cruzas Île de France e Texel, com peso vivo médio de 26 $\pm 4,80 \mathrm{~kg}$, e idade média de nove meses, que foram pesados, com jejum prévio de 12 horas, no início do experimento. As pesagens intermediárias foram feitas a cada ciclo de pastejo, para orientar o ajuste da lotação. Os animais tiveram acesso a sal mineralizado e água à vontade e controle sanitário periódico. O controle parasitário era feito através de exame OPG (ovos por grama de fezes) e quando necessário eram desvermifugados com princípios ativos específicos para cada espécie helmíntica segundo a coprocultura.

Os tratamentos consistiram de duas intensidades de pastejo (moderada e baixa) e dois métodos de pastejo (lotação contínua, $C$ e rotacionada, $R$ ), em três repetições. A intensidade de pastejo moderada (IPM) foi definida por uma oferta de forragem de 2,5 vezes o potencial de consumo dos animais (conforme o NRC, 1985), e a intensidade de pastejo baixa (IPB) por uma oferta de forragem de 5 vezes o potencial de consumo.

Para manter as ofertas pretendidas, foi utilizado um número variável de animais reguladores, através da técnica put-andtake (Mott e Lucas, 1952). Em razão da necessidade de se obter a mesma oferta de forragem para os dois métodos, o período de ajuste da carga para ambos foi o mesmo. A definição deste período foi feita utilizando-se os ciclos de pastejo da lotação rotacionada.

O tempo de duração de vida da folha (DVF) do azevém foi utilizado para determinação dos ciclos de pastejo, que permitiu definir o período de descanso a partir de uma variável morfogênica indicadora do intervalo ótimo de desfolhação. A DVF do azevém foi definida por Pontes et al. (2003): $500^{\circ} \mathrm{C} /$ folha, no período de junho a agosto e $410^{\circ} \mathrm{C} /$ folha, de setembro a novembro. Os ciclos de pastejo foram definidos dividindose a DVF do azevém pela temperatura média de cada um dos meses do período do experimento (julho a novembro). O resultado desse cálculo indicou quatro ciclos de pastejo com 35, 34, 24 e 22 dias, respectivamente. O período de ocupação das faixas foi de 2 dias.

A pastagem foi utilizada pelos cordeiros de 12 de julho a 1 de novembro de 2003 (113 dias de utilização). No início de outubro, quando a imposição dos tratamentos originou estruturas de pasto características aos manejos contrastantes de intensidades e métodos de pastejo, as avaliações foram feitas nos dias 6 e 7 de outubro, ocasião em que as temperaturas mínimas verificadas foram de 16,8 e $16,6^{\circ} \mathrm{C}$ e as máximas de 20,9 e $22,8^{\circ} \mathrm{C}$, respectivamente.

A massa de forragem (MF) foi avaliada no dia 3 de outubro, utilizando-se um disco medidor de forragem (Bransby et al., 1977) em 50 pontos no método de lotação contínua. Posteriormente foram feitos quatro cortes ao nível do solo, com o auxilio de um quadro metálico de $0,5 \times 0,5 \mathrm{~m}$. Nos piquetes de lotação rotacionada foram avaliadas as massas de forragem pré e pós-pastejo. Foram realizados dois cortes em uma faixa de pastejo do início do ciclo e dois cortes em outra faixa no final do ciclo de pastejo. A massa de forragem era obtida através da média dos cortes avaliados e os valores extrapolados para hectare. Todas as amostras de massa de forragem foram secas a $65^{\circ} \mathrm{C}$ por $72 \mathrm{~h}$, para determinação da matéria seca.

A altura da pastagem foi medida como sendo a distância entre o solo e a lâmina foliar mais elevada na superfície do pasto, usando-se um bastão graduado (sward stick) com medição em centímetros (Barthram, 
1985), no dia 3 de outubro. Foram feitas 50 avaliações nos piquetes de lotação contínua e 10 avaliações nos piquetes de lotação rotacionada.

Para a medição da taxa de acúmulo, no método lotação contínua, utilizou-se gaiolas de exclusão de pastejo (Klingman et al., 1943), com intervalo de avaliação de 21 dias (quatro gaiolas por unidade experimental). Nos piquetes de lotação rotacionada a taxa de acúmulo foi avaliada a cada ciclo de duração de vida da folha. No final de cada ciclo, amostras de forragem da segunda e da penúltima faixa de pastejo foram cortadas e a taxa de acúmulo foi obtida pela diferença dos valores dos cortes de massa de forragem residual.

A oferta de forragem foi calculada usando a seguinte fórmula:

Onde:

$$
O F=(M F / n+T A C) * 100 / C A
$$

$\mathrm{OF}=$ oferta de forragem (\%);

$\mathrm{MF}=$ massa de forragem média de cada ciclo de pastejo (kg de MS/ha);

$\mathrm{n}=$ número de dias do ciclo de pastejo (dias);

$\mathrm{TAC}=$ taxa de acúmulo da forragem (kg de MS/ha/ dia);

$\mathrm{CA}=$ carga animal média do ciclo de pastejo ( $\mathrm{kg}$ de $\mathrm{PV} / \mathrm{ha}$ ).

Foram observados três cordeiros em cada unidade experimental, identificados com marcas coloridas para facilitar a observação, do nascer ao pôr do sol. Para as avaliações das atividades tempo de pastejo (TP), tempo de ruminação (TR) e tempo para outras atividades (TO), foi utilizado o método direto de observação visual (Hughes e Reid, 1951) realizada a cada 10 minutos, por observadores previamente treinados.

O número de refeições (NR), o tempo de duração das refeições (TRf), o intervalo entre refeições (IR) e a duração do intervalo entre refeições (DMI), foram obtidos a partir do controle do tempo de pastejo (Penning e Rutter, 2004). Nesse trabalho, uma refeição é caracterizada a partir do momento em que o animal inicia o pastejo e permanece nessa atividade por no mínimo duas observações sucessivas (20 minutos). Uma vez decorrido este período, a refeição se caracteriza pelo tempo que o animal passa pastejando, até o momento em que atinge um determinado nível de ingestão e interrompe o pastejo, também por um período mínimo de 20 minutos. Esse período sem pastejo caracteriza um intervalo entre refeições.

Para a medida da taxa de bocados (TB) procederam-se estimativas visuais, registrando-se o tempo, em segundos, gasto pelos animais para dar 20 bocados (Forbes e Hodgson, 1985), sempre que fosse verificada atividade de pastejo. Este valor foi posteriormente transformado para número de bocados por minuto.

Para a determinação do tempo de cada estação alimentar (TEA), observou-se o tempo necessário à procura e utilização de 10 estações, de forma visual, semelhante ao procedimento para avaliação da taxa de bocados. Uma estação alimentar é definida como a área em que o animal pasteja sem movimentar as patas dianteiras (Ruyle e Dwyer, 1985).

Utilizou-se, para análise do comportamento ingestivo as médias obtidas em dois dias do período de ocupação no método de pastejo de lotação rotacionada, e de um dia no método de lotação contínua, assumindo-se que as ofertas de forragem eram comparáveis em qualquer dia dentro do mesmo ciclo de pastejo, no caso de lotação contínua, e em qualquer dos dois dias de ocupação, no caso da lotação rotacionada. Os dados obtidos foram submetidos à análise de variância (Proc GLM) e as médias foram comparadas pelo teste $\mathrm{F}$ a $10 \%$ de probabilidade, através do pacote estatístico SAS (1993). Utilizou-se o LSMEANS para efeito de comparação das médias entre tratamentos.

\section{RESULTADOS}

A caracterização da pastagem no período da avaliação do comportamento ingestivo é apresentada na tabela I. As OFs não

Archivos de zootecnia vol. 59, núm. 225, p. 42. 
foram diferentes ( $p=0$,3393) entre os métodos de pastejo, garantindo, assim, a condição fundamental para comparação entre os tratamentos. Não houve interação $(p>0,1)$ entre métodos e intensidades de pastejo. Assim sendo, seus efeitos foram analisados de forma independente.

A MF média dos tratamentos de IPM foi menor em relação aos de IPB ( $p=0,0015)$. Comparando-se a MF nos métodos de pastejo observa-se que, em média, esta variável foi maior nos tratamentos de pastejo de lotação rotacionada ( $p=0,0250)$.

As alturas do pasto na IPB foram maiores que na IPM ( $p=0,0001)$, enquanto que para métodos de pastejo, na lotação rotacionada as alturas foram maiores que na contínua $(\mathrm{p}=0,0002)$.

Todas as variáveis do comportamento ingestivo em pastejo são apresentadas na tabela II. A ausência de diferenças significativas para TP $(\mathrm{p}=0,3995), \mathrm{TR}(\mathrm{p}=0,6837)$, TO $(p=0,2626), N R(p=0,8381)$, TRf $(p=$ $0,2828), \operatorname{IR}(p=0,4993)$ eDMI ( $p=0,4257)$, em relação aos métodos de pastejo, significa que essas variáveis não foram influenciadas pela forma de condução do pastejo.

Foi registrado um tempo maior para a atividade de pastejo (TP) na IPM ( $p=0,0365)$. OTP foi correlacionado negativamente com a altura $(r=-0,73 ; p=0,006)$. Os cordeiros que estavam em baixa intensidade de pastejo passaram mais tempo em outras atividades do que os cordeiros que foram mantidos em intensidade de pastejo moderada $(p=0,0355)$.

Não houve diferença para TR $(p=0,3603)$ e NR ( $p=0,9413)$, nas diferentes intensidades de pastejo. A duração da refeição não diferiu entre os tratamentos $(p=0,2828$ para métodos e $\mathrm{p}=0,1342$ para intensidades) (tabela II). Não houve diferença entre métodos $(p=0,4993)$, nem entre intensidades de pastejo ( $\mathrm{p}=0,6512)$, para o número de intervalos entre refeições (IR), entretanto, a duração média do intervalo (DMI) foi menor para a IPM (p=0,0881).

A taxa de bocados (tabela II) foi menor na IPB ( $\mathrm{p}=0,0306)$ do que na IPM (34 versus 43 bocados/min.). Na lotação contínua, a taxa de bocados foi de 44,1 bocados/min., maior $(p=0,0030)$ do que na lotação rotacionada em que os cordeiros apresentaram uma taxa de 31,9 bocados/min. A TB foi negativamente correlacionada com a altura da pastagem $(\mathrm{p}=0,0065 ; \mathrm{r}=-0,73)$ e positivamente com o TP $(p=0,0017 ; r=0,80)$.

OTEA (tabela II) foi mais alto, tanto para IPM, quanto para a lotação rotacionada ( $\mathrm{p}=$ 0,0049 ). O TEA foi significativamente correlacionado com OF, MF e altura $(p=$

Tabela I. Parâmetros da pastagem do Lolium multiflorum; médias do período da avaliação de comportamento ingestivo em pastejo. (Parameters of Lolium multiflorum pasture, during the period of the evaluation of the ingestive behavior of the lambs).

\begin{tabular}{|c|c|c|c|c|c|c|c|}
\hline & $\begin{array}{r}\text { Intensid } \\
\text { de pas } \\
\text { moderada }\end{array}$ & $\begin{array}{l}\text { ades } \\
\text { bajo } \\
\text { baixa }\end{array}$ & $\begin{array}{l}\text { Valor } \\
\text { de } p\end{array}$ & $\begin{array}{l}\text { Método } \\
\text { lotação } \\
\text { contínua }\end{array}$ & $\begin{array}{l}\text { de pastejo } \\
\text { lotação } \\
\text { rotacionada }\end{array}$ & $\begin{array}{l}\text { Valor } \\
\text { de } p\end{array}$ & EP \\
\hline Oferta de forragem ${ }^{1}$ & 16 & 24 & $0,0051^{*}$ & 21 & 19 & $0,3393^{\text {ns }}$ & 3,18 \\
\hline Massa de forragem ${ }^{2}$ & 1971 & 3514 & $0,0015^{\star}$ & 2327 & 3159 & $0,0250 *$ & 485,74 \\
\hline Massa de lâminas² & 530 & 595 & $0,7402^{\mathrm{ns}}$ & 579 & 538 & $0,6220^{\text {ns }}$ & 114,33 \\
\hline Altura (cm) & 9 & 18 & $0,0001^{*}$ & 8 & 19 & $0,0002^{*}$ & 3,51 \\
\hline Carga animal $^{3}$ & 1448,76 & 1226,03 & - & 1110,39 & 1564,40 & - & - \\
\hline Lotação ${ }^{4}$ & 35,95 & 28,08 & - & 25,67 & 38,37 & - & - \\
\hline
\end{tabular}

${ }^{1} \mathrm{~kg}$ de MS/100 kg de PV/dia; ${ }^{2} \mathrm{~kg}$ de MS/ha; ${ }^{3} \mathrm{~kg} \mathrm{PV} / \mathrm{ha} ;{ }^{4}$ animais/ha.

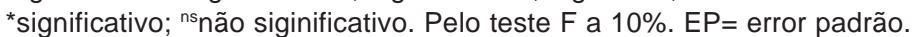


0,$0087 ; \mathrm{r}=0,71, \mathrm{p}=0,0059 ; \mathrm{r}=0,74 \mathrm{ep}<0,0001 ;$ $\mathrm{r}=0,89$, respectivamente), apresentando uma correlação negativa com o TP $(\mathrm{p}=0,0001$; $\mathrm{r}=-0,90)$.

\section{DISCUSSÃO}

A partir dos tratamentos impostos, optou-se por denominar as ofertas de forragem como baixa e moderada, uma vez que, para um ruminante a pasto desenvolver a sua máxima capacidade de ingestão, é necessário que seja oferecida uma quantidade de forragem, quatro vezes superior ao seu potencial de consumo (Gibb e Treacher, 1976). O potencial de consumo de um cordeiro, com as características dos animais no início do experimento, segundo o NRC (1985), era de 4\% do peso vivo. Conforme Silveira (2001) observou, em azevém, que uma oferta de matéria seca de 10,6\% do peso vivo resultou em certa restrição do consumo dos cordeiros e uma oferta de $18,9 \%$ do PV disponibilizou aos animais uma quantidade de forragem superior ao seu potencial de consumo. A partir desses dados, foi determinado como moderada intensidade de pastejo, 2,5 vezes o potencial de consumo ( $4 \%$ do peso vivo) e como baixa intensidade de pastejo, 5 vezes o potencial de consumo ( $4 \%$ do peso vivo). Como mostra a tabela I, os valores da oferta de forragem real ficaram muito próximos aos valores impostos.

A menor MF para IPM se deu em razão da menor oferta de forragem e, conseqüentemente, maior carga animal (1449 kg de PV/ ha versus 1226,03 kg de PV/ha). Pontes et al. (2003), também observaram que as menores alturas de condução do pasto foram obtidas com cargas animais maiores, resultando em menores valores de MF. Comparando-se a MF entre os métodos de pastejo, verificouse que seu valor foi maior no tratamento com lotação rotacionada em razão da freqüência de desfolha ter sido controlada em função do período de descanso pós-pastejo, característica esta inerente a este método de pastejo. É provável que o fato de dispor de

Tabela II. Médias das variáveis que compõem o processo ingestivo de cordeiros mantidos em pastagem de L olium multiflorum. (Mean of the variables of the ingestive process of lambs reared in Lolium multiflorum pastures).

\begin{tabular}{|c|c|c|c|c|c|c|c|}
\hline & $\begin{array}{l}\text { Intensid } \\
\text { de pas } \\
\text { noderada }\end{array}$ & $\begin{array}{l}\text { des } \\
\text { ejo } \\
\text { baixa }\end{array}$ & $\begin{array}{l}\text { Valor } \\
\text { de } p\end{array}$ & $\begin{array}{l}\text { Métodos } \\
\text { lotação } \\
\text { contínua }\end{array}$ & $\begin{array}{l}\text { de pastejo } \\
\text { lotação } \\
\text { rotacionada }\end{array}$ & $\begin{array}{l}\text { Valor } \\
\text { de } p\end{array}$ & EP \\
\hline \multicolumn{8}{|l|}{ Tempo de } \\
\hline pastejo (min.) & 531 & 455 & $0,0365^{\star}$ & 506 & 480 & $0,3995^{\text {ns }}$ & 49,02 \\
\hline ruminação (min.) & 167 & 181 & $0,3603^{\text {ns }}$ & 171 & 177 & $0,6837^{\mathrm{ns}}$ & 24,63 \\
\hline outras atividades (min.) & 87 & 147 & $0,2626 *$ & 103 & 131 & $0,2626^{\mathrm{ns}}$ & 38,06 \\
\hline \multicolumn{8}{|l|}{ Refeições } \\
\hline número & 4 & 4 & $0,9413^{\text {ns }}$ & 4 & 4 & $0,8381^{\mathrm{ns}}$ & 0,67 \\
\hline tempo médio (min.) & 139 & 111 & $0,1342^{\text {ns }}$ & 126 & 124 & $0,2828^{\text {ns }}$ & 37,07 \\
\hline intervalos entre $\left(n^{\circ}\right)$ & 3 & 3 & $0,6512^{\text {ns }}$ & 3,2 & 2,9 & $0,4993^{\text {ns }}$ & 0,80 \\
\hline duração do intervalo ${ }^{1}$ & 62 & 89 & $0,0881^{*}$ & 70 & 81 & $0,4257^{n s}$ & 23,23 \\
\hline Taxa de bocados (n\%/min.) & 42 & 34 & $0,0306 *$ & 44 & 32 & $0,0030 *$ & 4,41 \\
\hline Tempo da estação alimentar² & 28 & 11 & $0,0049 *$ & 8 & 10 & $0,0915^{\star}$ & 1,31 \\
\hline
\end{tabular}

${ }^{1}$ minutos; ${ }^{2}$ segundos.

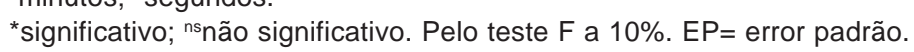

Archivos de zootecnia vol. 59, núm. 225, p. 44. 
um tempo programado para se recuperar, após o período de pastejo, tenha produzido uma estrutura com maior índice de área foliar nos tratamentos com lotação rotacionada, o que pode ser atestado pela maior MF nesses tratamentos. Este efeito foi discutido por Parsons et al. (2000), e é particularmente verdadeiro em situações de elevadas intensidades de pastejo.

O comportamento da variável altura foi similar ao da massa de forragem, quanto maior a intensidade de pastejo, menor a altura do pasto. Nos pastos mantidos sob lotação rotacionada há a programação de um período de rebrota, no qual a condição inicial de pós pastejo contrasta, em termos de fisiologia, com a condição de pré pastejo. As mudanças na estrutura do pasto são abruptas, num curto espaço de tempo, ocorrendo uma rápida depleção de forragem ao longo do período de ocupação, associada à nova condição do ambiente luminoso na condição de pós pastejo. Por outro lado, o manejo sob lotação contínua é caracterizado por uma estrutura de pasto mais estável ao longo do tempo.

Essas características de ambos os métodos, ao gerarem diferentes MF, influenciaram o tempo por estação alimentar (vide tabela II). A razão para este fato foi relatada por Prache e Peyraud (1997), segundo os quais a altura do pasto e a distribuição espacial das lâminas foliares são características que afetam a facilidade de coleta pelo animal. Uma vez que tanto a altura, quanto a massa de forragem, foram superiores no tratamento de lotação rotacionada, o maior tempo por estação alimentar indica um padrão de resposta típico de situações de abundância de forragem. Esta resposta está de acordo com a previsão do modelo de Charnov (1976), segundo o qual, o tempo de pastejo na estação alimentar aumenta com a maior disponibilidade de forragem. Desta forma, o animal pastejaria de forma otimizada, pois a taxa de consumo é maximizada pelo abandono das estações alimentares, quando iguala-se à média da taxa de ingestão obtida nas estações alimentares precedentes (Carvalho etal., 1999).

O maior tempo para atividade de pastejo (TP), observado na intensidade moderada, pode ser justificado pela menor quantidade de massa de forragem existente nesse tratamento, bem como da menor altura do pasto. Isto está de acordo com os resultados encontrados por vários autores, que mostraram que à medida que a quantidade de forragem diminui, o tempo de pastejo aumenta, com a finalidade de tentar maximizar o consumo, embora o aumento deste tempo seja limitado (Penning, 1986; Hodgson, 1990; Prache e Peyraud, 1997; Iason et al., 1999). Os tempos de pastejo verificados encontramse dentro da amplitude descrita como aceitável por Hodgson et al. (1994). Seu aumento é uma estratégia que os animais dispõem para tentar compensar a diminuição da massa do bocado em situações de baixa disponibilidade de forragem. Os resultados de Silveira (2001), avaliando alturas de pastejo com ovinos, em azevém anual, variaram linearmente de 609,7 a 485 minutos/dia, da menor para a maior altura. Neste trabalho, em pastagens mantidas com IPM, os animais gastaram cerca de uma hora a mais em atividade de pastejo, se comparados a animais mantidos em IPB. O mesmo autor mostrou também que, à medida que a oferta de forragem aumentava, os animais reduziam o tempo de pastejo e aumentavam o tempo de ruminação. Neste trabalho houve diminuição do tempo de pastejo em IPB, mas não houve efeito significativo para o tempo de ruminação (TR). Este resultado pode estar ligado ao fato da avaliação ter sido diurna, visto que grande parte da atividade de ruminação acontece no período noturno, apesar dos resultados de Silveira (2001) também terem sido obtidos em avaliações diurnas. Outra interpretação para esse resultado é que, mesmo em intensidades de pastejo moderada, a oferta de forragem ainda era suficiente para permitir uma seleção pela fração folha, permitindo que o tempo de ruminação não fosse alterado de forma sig- 
nificativa. O ajuste comportamental de pequenos ruminantes em resposta à baixa disponibilidade de alimento é, principalmente, manter a qualidade da dieta, enquanto grandes ruminantes mantêm a taxa de ingestão de matéria seca (Demment e Geenwood, 1988; Hodgson et al., 1991; Dumont et al., 1995). Entretanto, o tempo para outras atividades foi maior para os animais que estavam em baixa intensidade de pastejo. Pode-se dizer que, quando os cordeiros diminuíram o tempo de pastejo, gastaram mais tempo em outras atividades durante o dia, visto que essas atividades do comportamento ingestivo são excludentes (Carvalho et al., 2001; Trevisan et al., 2004).

Apesar do animal procurar compensar uma provável menor taxa de ingestão de matéria seca, com o aumento do tempo de pastejo na IPM, não houve diferença para NR nas diferentes intensidades. O número de refeições pode ser um indicador da qualidade do ambiente pastoril. A refeição termina com os animais tendo atingido um determinado nível de ingestão e, nesse ponto, um intervalo inter-refeições se inicia. Logo, em situações de elevadas massas de forragem, maior será o número de refeições e menor a duração de cada uma (Carvalho et al., 2005). É possível que a massa de forragem da IPM, embora menor quando comparada a IPB, não seria menor o suficiente para provocar alterações comportamentais de mais longo prazo, como é o caso do NR. De fato, a massa de forragem na IPM foi apenas ligeiramente inferior aquela (2104 kg de MS/ ha) que maximizou o desempenho de cordeiros em azevém, no experimento de Silveira(2001).

$\mathrm{Na}$ figura 1 pode-se visualizar a distribuição do tempo de pastejo ao longo do dia. Esse gráfico permite verificar que, no período da tarde, o tempo de pastejo foi maior do que durante a manhã, sendo que as últimas horas do dia são de pastejo quase que ininterrupto, ou seja, a duração da refeição é maior. Este resultado está de acordo com o reportado por Carvalho (1997), segundo o qual haveria uma concentração de atividades de pastejo no período da tarde, principalmente nas horas que antecedem o pôr do sol, coincidindo com elevados teores de carboidratos não estruturais e matéria seca nas plantas, o que seria um indicativo de otimização na estratégia de ingestão de nutrientes pelos animais. Nos tratamentos de pastejo de lotação rotacionada, em períodos que antecediam as trocas das faixas, tinha-se a impressão que os animais ficavam aguardando a troca, parecendo relutar em gastar mais tempo e energia selecionando pequenas quantidades de lâminas no meio do material morto e excrementos da massa de forragem residual. Desta maneira, após a troca das cercas, no fim da tarde, os animais eram também estimulados a iniciar um longo período de pastejo, a fim de otimizar a ingestão de nutrientes, confirmando a proposta do modelo de Baumont et al. (2004), que combina a arquitetura da pastagem e as decisões alimentares dos animais, com os mecanismos de controle de consumo (balanço energético, motivação para consumo e satisfação alimentar).

Os resultados para o IR corroboram parcialmente a afirmação de Silveira (2001), à medida que a altura do pasto e a massa de

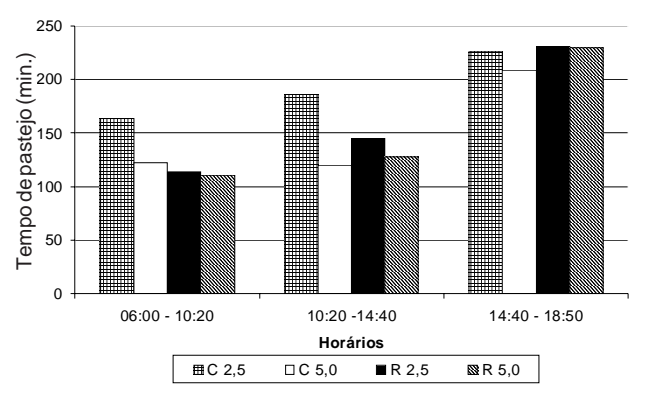

Figura 1. Distribuição ao longo do dia, do tempo de pastejo de cordeiros mantidos em pastagem de azevém anual (Lolium multiflorum) em duas intensidades e dois métodos de pastejo. (Distribution of the grazing time along the day of lambs reared under two intensities and two methods of grazing in ryegrass pastures (Lolium multiflorum).

Archivos de zootecnia vol. 59, núm. 225, p. 46. 
forragem aumentam, menor será a duração da refeição e maior será o número de intervalos entre refeições. Não houve diferença entre os tratamentos para esta última variável. Entretanto, a DMI foi maior para IPB do que para IPM, ou seja, em pastagens mais baixas os animais voltam mais rapidamente ao pastejo. Os trabalhos em comportamento ingestivo, já realizados, mostram que os animais alteram, tanto a duração, quanto o número dos processos, de forma diferente; em função do nível de restrição alimentar. Nesse estudo, na medida que se trabalhou com um nível de restrição alimentar apenas moderado, os animais operaram dentro de um nível intermediário de reação à condição do pasto. Tal restrição moderada estaria promovendo uma influência inicial no comportamento, via alteração do tempo das atividades conforme a tabela II. Todavia sem a ocorrência de mudança no número de atividades. A alteração no número de atividades representaria um segundo estágio de respostas comportamentais às restrições impostas pela pastagem, uma vez que o custo energético, para aumentar ou diminuir a duração da atividade do animal, é menor do que o custo de começar uma atividade nova. Os resultados apresentados nesse trabalho corroboram o que foi descrito por Kyriazakis (2003) e Carvalho et al. (2005). Os animais regulam o consumo dentro e entre refeições ao longo do dia, coordenando períodos de atividades de ingestão e de ruminação. As características das refeições (duração, número, distribuição ao longo do dia, etc.) e a magnitude da forragem ingerida, são reflexos diretos da qualidade, quantidade e estrutura do pasto que se oferece ao animal (Carvalho e Moraes, 2005).

A taxa de bocados foi semelhante às encontradas por vários autores (Hodgson, 1990; Castro, 2002; Trevisan et al., 2004) tendo os animais, em situação de baixa oferta de forragem, modificado suas atividades de pastejo. Em resposta as baixas ofertas de forragem, os animais aumentam a taxa de bocados, na medida em que mais movimentos mandibulares podem ser alocados para preensão da forragem (Carvalho et al., 2001). Os valores observados apresentaram uma amplitude semelhante aos de Freitas (2003), que obteve taxas variando de 43,6 a 62,6 bocados/min., e as citadas por Castro (2002), com resultados na faixa de 23,1 a 36,9 bocados/min. Carvalho (1997) sugeriu que, para a maioria dos herbívoros, existe um intervalo de 30 a 70 bocados/minuto. Em relação aos métodos de pastejo, a TB foi menor para a lotação rotacionada do que para a lotação contínua. Os valores de massa de forragem mais elevados para a lotação rotacionada explicam a diminuição da taxa de bocados nesse tratamento. Esse comportamento é atribuído ao aumento do tempo gasto para a manipulação e mastigação das lâminas foliares antes da deglutição das mesmas (Carvalho et al., 2001). O aumento na taxa de bocados e/ou no tempo de pastejo, com o objetivo de compensar a diminuição na oferta de forragem, foi confirmado por vários autores (Arnold, 1987; Hodgson, 1990; Carvalho et al., 2001), indicando que são estratégias de forrageamento adotadas pelos animais (Gordon et al., 1996). Entretanto, essa estratégia, não impede uma baixa ingestão de nutrientes, devido ao fato que o animal executa, ao longo do dia, diferentes atividades como ruminação, descanso e atividades sociais (Carvalho et al., 2001). Os valores mais altos de MF para a lotação rotacionada explicam também valores maiores para TEA, em relação aos métodos de pastejo (tabela II). Os pastos mais altos, com uma maior massa de forragem, proporcionam um elevado tempo de permanência em cada estação alimentar, sendo assim, no método de lotação contínua, as estações alimentares apresentaram um tempo de permanência menor, pois rapidamente atingiam o ponto de abandono. O TEA foi também influenciado pelas intensidades de pastejo, certificando que a menor freqüência de troca de estações alimentares, ou seja, o maior tempo de duração da estação alimen-

Archivos de zootecnia vol. 59, núm. 225, p. 47. 
tar, na IPB, é conseqüência da maior disponibilidade de forragem.

Considerando as características da vegetação, como influentes na escolha de estações alimentares, duas situações podem ser identificadas. Quando os animais podem pastejar em suas estações preferidas sem ter que buscá-las, ou quando a procura por estações alimentares modifica as preferências (Baumont et al., 2000). Esse comportamento está de acordo com as considerações feitas por Prache et al. (1998), segundo as quais, em condições de baixa disponibilidade de forragem, os animais caminham menos entre estações alimentares sucessivas, aumentando o número de estações alimentares visitadas e diminuindo o tempo de permanência nessas. O mesmo padrão de comportamento foi observado em novilhas em pastagens de capim Mombaça (Palhano et al., 2007), indicando que essa variável é consistente na indicação e diagnóstico da quantidade de alimento em oferta nos ecossistemas pastoris. Em maior disponibilidade de pasto, os animais caminham menos e elegem menor número de estações alimentares, entretanto, as exploram mais intensivamente, à medida que se caracterizam por maior oferta de alimento (Prache e Peyraud, 2001). Rook et al. (2004), encontraram valores médios menores que os obtidos neste trabalho, 5,3 segundos para duração da estação alimentar, com ovelhas pastejando azevém perene (Lolium perenne L.). Todas as atividades relacionadas ao comportamento ingestivo de

\section{BIBLIOGRAFIA}

Arnold, G.W. 1987.Grazing behaviour. In: Snaydon, R.W. (Ed.). Managed grassland analytical studies. Elsevier. Oxford. pp. 129-135.

Barthram, G.T. 1985. Experimental techniques: The HFRO sward stick. In: Hill Farming Research Organization/Biennial Report. HFRO. Aberdeen. pp. 29-30.

Baumont, R., Cohen-Salmon, D., Prache, S. and Sauvant, D. 2004. A mechanistic model of intake and grazing behaviour in sheep integrating ruminantes em pastejo estão distribuídas de maneira que o propósito de maximização do consumo de forragem pelo animal seja alcançado (Charnov, 1976). Desta forma, aumenta o desvio de energia líquida para mantença que junto com a menor qualidade nutricional, resulta em baixo desempenho animal. Entretanto, caso não houvesse aumento na seleção de folhas, a queda no desempenho poderia ainda ser maior.

É importante salientar que o conhecimento dessas respostas é de grande contribuição na busca de melhores condições de manejo, enaltecendo a magnitude de se criar ambientes pastoris adequados e compatíveis com os requerimentos dos animais.

\section{CONCLUSÕES}

Os métodos de pastejo não influenciaram o tempo de pastejo ao contrário das diferentes intensidades de pastejo. Altas taxas de bocado associadas a menores tempos de duração da estação alimentar são comportamentos, que contextualizam uma situação de insuficiência de alimento em pastagens. Em situações de restrição alimentar moderada, os animais apresentam estratégias alimentares compensatórias, visando aumentar sua ingestão de forragem.

$\mathrm{Na}$ construção de ambientes pastoris sustentáveis à produção de cordeiros, a intensidade de pastejo adequada ao desempenho dos animais é mais importante do que o método de pastejo.

sward architecture and animal decisions. Anim. Feed Sci.Tech., 112: 5-28.

Baumont, R., Prache, S. and Meuret, M. 2000. How forage characteristics influence behaviour and intake in small ruminants: a review. Livest. Prod. Sci., 64: 15-28.

Bransby, D.I, Matches, A.G. and Krause, G.F. 1977. Disk meter for rapid estimation of herbage yield in grazing trials. Agron. J., 69: 393-396. Carvalho, P.C.F. 1997. A estrutura da pastagem e

Archivos de zootecnia vol. 59, núm. 225, p. 48. 


\section{PASTEJO DE CORDEIROS EM AZEVÉM (LOLIUM MULTIFLORUM)}

o comportamento ingestivo de ruminantes em pastejo. Em: Simpósio sobre avaliação de pastagens com animais, 2. Anais... Universidade Estadual de Maringá. Ed. Maringá. Maringá. pp. 25-52.

Carvalho, P.C.F., Prache, S. e Damasceno, J.C. 1999. O processo de pastejo: desafios da procura e apreensão da forragem pelo herbívoro. Em: XXXVI Reunião Anual da Sociedade Brasileira de Zootecnia, 36. Anais... UFRGS. Porto Alegre. pp. 253-268.

Carvalho, P.C.F., Ribeiro Filho, H.M.N., Poli, C.H.E.C., Moraes, A. e Delagarde, R. 2001. Importância da estrutura da pastagem na ingestão e seleção de dietas pelo animal em pastejo. Em: Mattos, W.R.S. (Org.). A Produção Animal na visão dos brasileiros. Ed. FEALQ. Piracicaba. pp. 853-871.

Carvalho, P.C.F. 2005. O manejo da pastagem como gerador de ambientes pastoris adequados à produção animal. Em: Pedreira, C.G.S., Moura, J.C. de, Silva, S.C. da, Faria, V.P. de (Eds.) Teoria e prática da produção animal em pastagens. 1 ed. Ed. FEALQ. Piracicaba. pp. 7 32.

Carvalho, P.C.F. e Moraes, A. 2005. Comportamento ingestivo de ruminantes: bases para o manejo sustentável do pasto. Em: Ulysses Cecato; Clóves Cabreira Jobim. (Org.). Manejo sustentável em pastagem. vol 1. Ed. Maringá. Maringá. pp. 1-20.

Carvalho, P.C.F., Genro, T.C.M., Gonçalves, E.N. e Baumont, R. 2005. A estrutura do pasto como conceito de manejo: reflexos sobre o consumo e a produtividade. Em: Ricardo Andrade Reis; Gustavo Resende Siqueira; Liandra M.A. Bertipaglia; Amanda Prates Oliveira; Gabriel M.P. de Melo; Thiago Fernandes Bernardes. (Org.). II Simpósio de volumosos na produção de ruminantes. UNESP. Jaboticabal. pp. 107124.

Castro, C.R.C. 2002. Relações planta-animal em pastagem de milheto (Pennisetum clandestinum (L.) Leeke) manejadas em diferentes alturas com ovinos. Dissertação (Mestrado em Zootecnia). Universidade Federal do Rio Grande do Sul. 200 pp.

Charnov, E.L. 1976. Optimal foraging: the marginal value theorem. Theor. Popul. Biol., 9: 129-136.

Demment, M.W. and Geenwood, G.B. 1988. Forage ingestion: effects of sward characteristics and body size. Anim. Sci. J., 66: 2380-2392.

Dumont, B., Petit, M. and D'Hour, P. 1995. Choice of sheep and cattle between vegetative and reproductive cocksfoot patches. Appl. Anim. Behav. Sci., 43: 1-15.

Forbes, T.D.A. and Hodgson, J. 1985. Comparative studies of the influence of sward conditions on the ingestive behaviour of cows and sheep. Grass Forage Sci., 40: 69-77.

Freitas, T.M.S. 2003. Dinâmica da produção de forragem, comportamento ingestivo e produção de ovelhas Ile de France em pastagem de azevém anual (Lolium multiflorum Lam.) em resposta a doses de nitrogênio. Dissertação (Mestrado em Zootecnia). Universidade Federal do Rio Grande do Sul. 180 pp.

Gibb, M.J. and Treacher, T.T. 1976. The effect of herbage allowance on herbage intake and performance of lambs grazing perennial ryegrass and red clover swards. J. Agric. Sci., 86: 355365.

Gordon, I.J., Illius, A.W. and Milne, J.D. 1996. Sources of variation in the foraging efficiency of grazing ruminants. Funct. Ecol., 10: 219-226.

Hodgson, J. 1990. Grazing management: Science into practice. Longman Handbooks in Agriculture. John Wiley \& Sons, Inc. New York. pp. 25-37. Hodgson, J., Clark, D.A. and Mitchell, R.J. 1994. Foraging behavior in grazing animals and its impact on plant communities. In: Fahey, G.C. (Ed.). Forage quality and utilization. Based on the National Conference on Forage Quality. American Society of Agronomy. Lincon. pp. 796-827.

Hodgson, J., Forbes, T.D.A., Armstrong, R.H., Beattie, M.M. and Hunter, E.A. 1991. Comparative studies of the ingestive behaviour and herbage intake of sheep and cattle grazing indigenous hill plant communities. J. Appl. Ecol., 28: 205-227.

Hughes, G.P. and Reid, D. 1951. Studies on the behavior of cattle and sheep in relation to utilization of grass. J. Agric. Sci., 41: 350-355. Iason, G.R., Mantecon, A.R., Sim, D.A., Gonzalez, J., Foreman, E., Bermudez, F.F. and Elston, D.A. 1999. Can grazing sheep compensate for a daily foraging time constraint? J. Anim. Ecol., 68: 87-93

Klingman, D.L., Miles, S.R. and Mott, G.O. 1943. The cage method for determine consumption and yield of pasture herbage. J. Am. Soc.

Archivos de zootecnia vol. 59, núm. 225, p. 49. 


\section{BARBOSA, CARVALHO, CAUDURO, LUNARDI, GONÇALVES E DEVINCENZI}

Agron., 35: 739-746.

Kyriazakis, I. 2003. What are ruminant herbivores trying to achieve through their feeding behaviour and food intake? In: VI International Symposium on the Nutrition of Herbivores. Proceedings... Universidad Autónoma de Yucatán. Mérida. pp. 154-173.

Mott, G.O. and Lucas, H.L. 1952. The design, conduct, and interpretation of grazing trials on cultivated and improved pastures. In: International Grassland Congress, 6. Pensylvania Proceedings... pp. 1380-1385.

NRC. 1985. Nutrient requirement of sheep. $6^{\mathrm{a}} \mathrm{ed}$. Ed. National Academy of Science. USA. 99 pp.

Palhano, A.L., Carvalho, P.C.F., Dittrich, J.R., Moraes, A., Silva, S.C. e Monteiro, A.L.G. 2007. Características do processo de ingestão de forragem por novilhas holandesas em pastagens de capim-mombaça. Rev. Bras. Zootecn., 36: 1014-1021.

Parsons, A.J., Carrère, P. and Schwinning, S. 2000. Dynamics of heterogeneity in a grazed sward. In: Lemaire, G.; Hodgson, J.; Moraes, A.; Carvalho, P.C.F.; Nabinger, C. (Org.). Grassland Ecophysiology and Grazing Ecology. Ed. CABI Publishing. Wallingford-Oxon. v. 1. pp. 289-316.

Penning, P.D. and Rutter, S.M. 2004. Ingestive behaviour. In: Penning, P.D. (ed.). Herbage Intake Handbook - $2^{\text {nd }}$ ed. Chapter 6. British Grassland Society. Reading. pp. 151-175.

Penning, P. 1986. Some effects of sward conditions on grazing behaviour and intake by sheep. In: Gudmundssun, O. (Ed.). Grazing research at Nothern Latitudes. Ed. Plenum Press. New York. pp. 219-226.

Pontes, L.S., Nabinger, C., Carvalho, P.C. de F., Trindade, J.K., Montardo, D.P. e Santos, R.J. 2003. Variáveis morfogênicas e estruturais de azevém anual (Lolium multiflorum Lam.) mane- jado em diferentes alturas. Rev. Bras. Zootecn. 32: 814-820.

Prache, S. et Peyraud, J. 1997. Préhensibilité de I'herbe pâturée chez les bovins et les ovins. Prod. Anim., 10: 377-390.

Prache, S. and Peyraud, J. 2001. Foraging: behaviour and intake in temperate cultivated grassland. In: International Grassland Congress, 19. Proceedings... São Pedro. São Pedro. pp. 309-319.

Prache, S., Roguet, C. and Petit, M. 1998. How degree of selectivity modifies foraging behaviour of dry ewes on reproductive compared to vegetative sward structure. Appl. Anim. Behav. Sci., 57: 91-108.

Rook, A.J., Harvey, A., Parsons, A.J., Orr, R.J. and Rutter, S.M. 2004. Bite dimensions and grazing movements by sheep and cattle grazing homogeneous perennial ryegrass swards. Appl. Anim. Behav. Sci., 88: 227-242.

Ruyle, G.B. and Dwyer, D.D. 1985. Feeding stations of sheep as an indicator of diminished forage supply. J. Anim. Sci., 51: 349-353.

SAS. 1993. SAS/STAT user's guide: statistics. 4 ed. Version 6. Statitical Analysis System. SAS Institute Inc. Cary, NC. v. 2. 943 pp.

Silveira, E.O. 2001. Produção e comportamento ingestivo de cordeiros em pastagem de azevém anual (Lolium multiflorum Lam.) manejada em diferentes alturas. Tese (Doutorado em Zootecnia). Universidade Federal do Rio Grande do Sul. 228 pp.

Trevisan, N.B., Quadros, F.L.F. de, Silva, A.C.F da, Bandinelli, D.G., Martins, C.E.N., Simões, L.F.C., Maixner, A.R. e Pires, D.R.F. 2004. Comportamento ingestivo de novilhos de corte em pastagem de aveia preta e azevém com níveis distintos de folhas verdes. Cienc. Rural, 34: 1543-1548.

Archivos de zootecnia vol. 59, núm. 225, p. 50. 